\title{
Optimizing Cursor Movement in Holistic Twig Joins
}

\author{
Marcus Fontoura, Vanja Josifovski, Eugene Shekita, and Beverly Yang \\ IBM Almaden Research Center \\ San Jose, CA, USA \\ trevi@almaden.ibm.com
}

\begin{abstract}
Holistic twig join algorithms represent the state of the art for evaluating path expressions in XML queries. Using inverted indexes on XML elements, holistic twig joins move a set of index cursors in a coordinated way to quickly find structural matches. Because each cursor move can trigger I/O, the performance of a holistic twig join is largely determined by how many cursor moves it makes, yet, surprisingly, existing join algorithms have not been optimized along these lines. In this paper, we describe TwigOptimal, a new holistic twig join algorithm with optimal cursor movement. We sketch the proof of TwigOptimal's optimality, and describe how TwigOptimal can use information in the return clause of XQuery to boost its performance. Finally, experimental results are presented, showing TwigOptimal's superiority over existing holistic twig join algorithms.
\end{abstract}

Categories and Subject Descriptors: H.3[Information Systems]: Information Storage and Retrieval; E.1[Data]: Data Structures

General Terms: Algorithms, Performance, Experimentation

Keywords: XML, Twig Joins, Indexing, Evaluation

\section{INTRODUCTION}

Both XPath and XQuery allow users to specify value and structural constraints in XML queries using path expressions. A path expression can be represented as a query tree, which is structurally matched against XML data. Performing this structural matching as efficiently as possible is one of the key issues in building an XML query engine.

There are two common approaches to perform structural matching efficiently. One approach is to use a structural join $[1,5,19]$, where a query tree is decomposed into a set of binary ancestor-descendant or parent-child relationships. The relationships are then evaluated using a binary merge join. Another approach is to use a holistic twig join [3, 10], which processes a query tree with a single n-ary join.

Holistic twig joins represent the state of the art for eval-

Permission to make digital or hard copies of all or part of this work for personal or classroom use is granted without fee provided that copies are not made or distributed for proft or commercial advantage and that copies bear this notice and the full citation on the frst page. To copy otherwise, to republish, to post on servers or to redistribute to lists, requires prior specifc permission and/or a fee.

CIKM'05, October 31-November 5, 2005, Bremen, Germany.

Copyright 2005 ACM 1-59593-140-6/05/0010 ...\$5.00. uating path expressions in XML queries. Not only do they perform better [10], but they are self-tuning and do not require a query optimizer. Holistic twig joins are index-based, typically relying on an inverted index for positional information about XML elements. Cursors are used to access the inverted index and moved in a coordinated way to efficiently find structural matches.

Several variations on holistic twig joins have been proposed in the literature $[3,4,9,10,16,18]$. Because each cursor move in an inverted index can trigger $\mathrm{I} / \mathrm{O}$, the performance of a twig join ${ }^{1}$ is largely determined by how many cursor moves it makes. Despite this observation, existing twig join algorithms have not been optimized along these lines. There has been more focus on minimizing the memory requirements of intermediate results than on minimizing the number of cursors moves. The problem with existing twig join algorithms is that they make a local, and hence sub-optimal, decision in choosing which cursor to move next and how far to move it.

In this paper, we describe TwigOptimal, a new holistic twig join algorithm with optimal cursor movement. This is accomplished by looking more globally at the query's state to determine which cursor to move next. We provide a sketch of TwigOptimal's optimality, and present experimental results, showing its superiority over existing twig join algorithms.

Another shortcoming of existing twig join algorithms is that they assume all nodes in a query tree need to be output. However, an XQuery return clause often requires only a subset of the nodes being matched to be output. We refer to the nodes that need to be output in the return clause as extraction points. By being aware of extraction points, we show how TwigOptimal can dramatically reduce the number of cursor moves it makes. This is accomplished by skipping over nodes that do not need to be output.

In summary, the main contributions of this paper are:

- A new holistic twig join algorithm called TwigOptimal that handles and-or branches and outperforms existing algorithms by optimizing its cursor movement.

- A description of how XQuery extraction points can be used to further improve TwigOptimal's performance.

- Experimental results showing TwigOptimal's superiority over existing holistic twig join algorithms.

\footnotetext{
${ }^{1}$ Note that, for readability, we will often use "twig join" as a shorthand for "holistic twig join".
} 


\section{RELATED WORK}

Structural joins $[1,5,19]$ were the first method proposed for evaluating path expressions. In a structural join, complex path expressions are broken down into a set of ancestordescendant or parent-child relationships and then evaluated using binary merge joins.

Holistic twig joins were first described in [3], which showed how performance could be improved by considering all nodes in the query tree holistically. Optimizations to the original twig join algorithm were later described in [10] and then extended to handle OR predicates in [9]. Several papers have also investigated new index types to speed up twig joins $[5,8,10,12,18])$. TwigOptimal provides the same functionality offered by existing twig joins, including OR predicates. Although a standard inverted index is assumed in this paper, TwigOptimal can also be used with many of the new index types that have been proposed to speed up twig joins.

Reference [10] is the only other paper that really addresses the problem of choosing which index cursor to move next in a holistic twig join. In [10], when the cursor positions do not form a structural match, a "broken edge" in the query tree is chosen using various heuristics and then "fixed". Fixing a broken edge consists of repeatedly moving the two cursors forming the edge until they structurally match the query. The problem with this approach is that, once a broken edge is chosen, only the two cursors forming the edge can be moved. In contrast, using the concept of virtual cursor moves, TwigOptimal is able to consider the whole query's cursor state every time a cursor needs to be moved.

To evaluate a path expression like $/ \mathrm{x} / \mathrm{y} / \mathrm{z}$, a holistic twig join will typically open three index cursors, one for each path step. However, if the position of data nodes are encoded with a Dewey value [15], the cursors for $x$ and $y$ can be derived from the cursor for $z$. In [18], these derived cursors were called virtual cursors. The choice of names is perhaps unfortunate, but the virtual cursors described in [18] should not be confused with the virtual cursor moves described in this paper. The former describes a derived cursor, whereas the latter describes a virtual move of a physical cursor.

References [2] and [11] described methods for minimizing the memory requirements of intermediate results when processing XQuery fragments over XML streams. Similarly, $[3,10]$ described methods for minimizing the memory requirements of intermediate results in twig joins. However, the performance of a twig join is largely determined by how many cursor moves it makes. Therefore, the focus in this paper is on minimizing the number of cursor moves in a twig join rather than on its memory requirements.

\section{BACKGROUND}

In this section, we establish some background that will be used in the remainder of the paper.

\subsection{Query Trees}

As in the previous work on holistic twig joins [3, 10], we will focus on XPath expressions or XQuery fragments that can be represented by a single query tree and structurally matched against XML data in one pass over an index. These are basically single-document path expressions containing child ('/') axis, descendant ('//') axis, and equality predicates, all of which can be combined using Boolean $A N D$ and $O R$ operators.

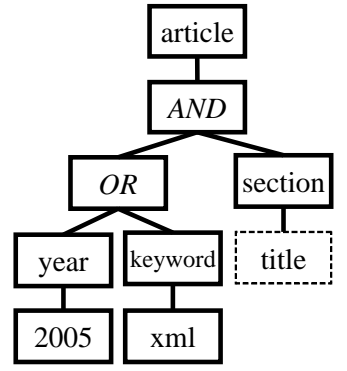

Figure 1: Example query tree

As an example, consider the following XQuery fragment, which returns the titles of XML articles in 2005:

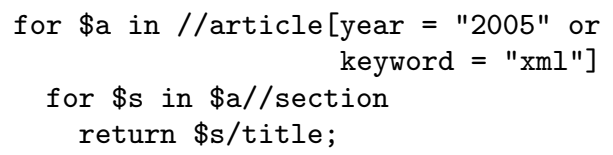

This query has three XPath expressions, one in each of the two for clauses, and one in the return clause. Figure 1 shows the resulting query tree. Each XPath step in Figure 1 is represented by a path node. There are also $A N D$ and $O R$ branching nodes, which specify that all or at least one of the subpatterns below the node must be matched, respectively. Finally, dotted lines are used to indicate that a path node corresponds to an extraction point, i.e., it is returned by the query. In general a query tree can have more than one extraction point since the for-let-where block of XQuery returns tuples of bindings.

\subsection{Inverted Indexes}

XML data is also commonly represented as a tree, with nodes corresponding to text values, elements, or attributes, and edges capturing the nesting of elements. The position of XML data nodes are used by holistic twig joins to do structural matching. The position of an XML data node can be encoded in different ways. One alternative is to use the well-known BEL encoding, where a node's begin, end, level forms its position. Another alternative is the Dewey encoding [15]. In this paper, we assume BEL encoding, but the techniques we describe are orthogonal to the choice of the encoding.

Holistic twig joins have been implemented over several indexing structures $[8,13,16]$. Here, we assume a standard inverted index [7]. Inverted indexes have stood the test of time and are frequently used in information retrieval and XML systems alike [1, 3, 5]). Briefly, an inverted index consists of one posting list per distinct token in the dataset, where a token can be a text value, attribute, or element tag. A posting list contains one posting for each occurrence of its token in the dataset and is sorted by position (in our case using BEL). Stepping through the posting list for a token $T$ will enumerate the positions of every occurrence of $T$ in order, by document and then within document. We assume each posting list is itself indexed, typically with a B-tree, so that searching for a particular position within a posting list is efficient.

\subsection{Query Evaluation}

To find documents that structurally match a query tree $Q$, a holistic twig join associates each path node $q$ with the posting list in the inverted index whose token matches $q$. 
A cursor is opened for each of these posting lists, and then moved in a coordinated way to find documents that structurally match $Q$. Parent-child and ancestor-descendant constraints imposed by the query are checked by looking at the current positions of cursors. The output of a holistic twig join is a stream of tuples, where each tuple corresponds to a solution, that is, a set of data nodes that structurally match the path nodes in $Q$. In existing twig join algorithms, all possible solutions are output $[3,10]$.

\section{THE TWIGOPTIMAL ALGORITHM}

In this section we describe TwigOptimal. For simplicity and to make the comparison to existing algorithms clearer, TwigOptimal is initially described without taking extraction points into account. Extraction points are addressed in Section 7 .

\subsection{Data Structures}

As in existing holistic twig join algorithms [3, 10], the evaluation state of TwigOptimal is a triplet $\langle Q, C, S\rangle$, where $Q$ is the query tree being evaluated, $C$ is the set of cursors for accessing the inverted index, and $S$ is a set of stacks for constructing solutions. Data nodes that are part of a solution are stacked on $S$ and output when a full solution is found.

An index cursor $C q$ and a stack $S q$ are associated with each path node $q$ in $Q$. $C q$ points to the current posting for $q$, while $S q$ is used to remember the data nodes for $q$ that are part of a solution. Each stack entry also has a pointer to an entry in an ancestor stack, which is used by TwigOptimal to output a solution, much like in $[3,10,11]$.

The position of a cursor $C q$ is accessed via Cq.begin, Cq.end, and Cq.level, and similarly for a stacked node in $S q$. Parent-child or ancestor-descendant constraints are checked by looking at BEL values. We say a cursor $C p$ contains another cursor $C q$ iff $C p$.begin $\leq C q$.begin and Cp.end $\geq$ Cq.end. Similarly, we say that a stack $S p$ contains $C q$ if there is some entry in $S p$ that contains $C q$.

For each cursor $C q$, the method $C q$. forwardTo(pos) moves $C q$ forward from its current position to the first position greater than or equal to pos. This can trigger I/O as $C q$ physically seeks to pos. To optimize its cursor movements, TwigOptimal also uses virtual cursor moves that, unlike physical cursor moves, do not trigger I/O. Instead, a virtual move on $C q$ simply sets $C q$.begin without physically moving $C q$. This will be made clearer shortly. Cq.virtual is set to true whenever $C q$ is virtually moved and reset to false whenever $C q$ is physically moved.

Unlike path nodes, each branching node in $Q$ is not associated with a posting list in the inverted index. However, each branching node does have a cursor, which is used to pass along the position of its parent or a child cursor when TwigOptimal is deciding which cursor to move next. Consequently, the cursor of a branching node is always virtual. By maintaining a cursor for each branching node, TwigOptimal does not need to distinguish between path nodes and branching nodes in most cases, which in turn simplifies the algorithm.

\subsection{The Main Loop}

ExecuteQuery(), which is shown in Figure 2, forms the entry point and main loop of TwigOptimal. It initializes each cursor to their first posting (line 1), then it repeatedly inspects the path node $q$ corresponding to the min cursor (line 3), that is, the cursor with the smallest begin value, until is has found and output all solutions.

To find and output solutions, the cursors are moved until

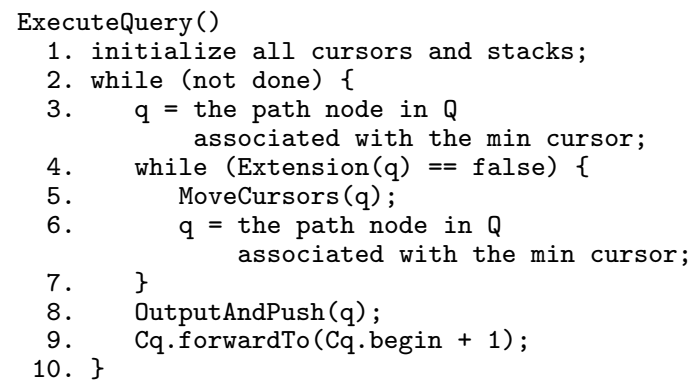

Figure 2: The main loop

an extension [10] for $q$ is found (lines 4-7). An extension for $q$ is basically a partial solution rooted at $q$. When an extension for $q$ has been found, the cursor positions in the subtree rooted at $q$ are guaranteed to be a part of a solution. In addition, the stacks of $q$ 's ancestors contain the position of data nodes that, when combined with the extension for $q$, forms a full solution.

Once an extension has been found, OutputAndPush() is called (line 8) to output any new stacked solutions and push $C q$ onto its stack. As in [10], $C q$ is only stacked when it is part of a solution. Finally, $C q$ is advanced to its next physical location (line 9) to start the search for another solution. The main loop terminates when the end of one or more posting lists is reached, allowing the algorithm to conclude that no more solutions can be found.

\subsection{Checking an Extension}

Extension(), which is shown in Figure 3, checks whether the cursor positions in the subtree rooted at $q$ form an extension. $\{\mathrm{C}\}$ is the set path of cursors in the subtree rooted at $q$. For the subtree rooted at $q$ to form an extension, $C q$ must be contained by its parent's stack $S p$, all the cursors in $\{C\}$ must be real (not virtual), and all the cursors in $\{C\}$ must recursively satisfy the containment constraints of $\mathrm{Q}$ (line 3). In the latter case, a cursor with an AND under it needs to contain all its children cursors, while a cursor with and OR under it needs to contain at least one of its children cursors.

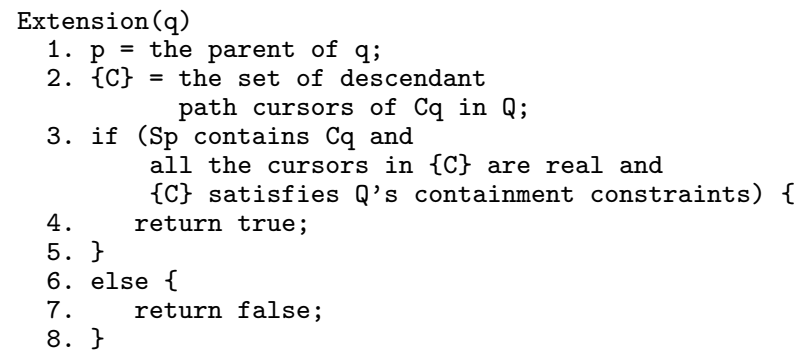

Figure 3: Checking an extension

\subsection{Moving the Cursors}

TwigOptimal calls MoveCursors(q) in its main loop as it searches for an extension, where $q$ corresponds to the min cursor. MoveCursors() basically tries to move the cursors in the subtree rooted at $q$ to the next extension for $q$, if any. To avoid I/O, this is done using virtual cursor moves. A physical cursor move is made only when further virtual progress becomes impossible.

MoveCursors() is shown in Figure 4. Two passes over the subtree rooted at $q$ are made to virtually move the cursors, a bottom-up pass (line 6) and a top-down pass (line 
7). The two passes over the subtree globally discover the furthest each cursor can be moved forward without missing an extension for $q$. This is in contrast to existing twig join algorithms, where cursors are moved in a localized way, only looking at the positions of one parent-child pair of cursors at a time.

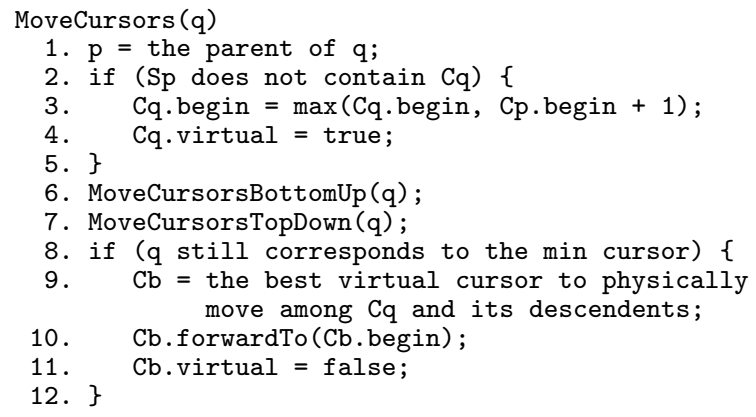

Figure 4: Moving the cursors

MoveCursors() begins by checking whether $C q$ is contained by its parent stack (line 2 ). If not, then $C q$ is virtually moved to the max of $C q$.begin or $C$ p.begin +1 , which is the most $C q$ can be moved forward without missing an extension for $q$. Next, the cursors are virtually moved bottom-up and then top-down (lines 6-7), as described earlier. Finally, a check is made to see whether $q$ still corresponds to the min cursor (line 8). If so, then further virtual progress is impossible, at which point a physical cursor move is made (lines 9-11). Note that, on exit, MoveCursors() may or may not have actually found an extension for $q$. This is checked in the main loop of TwigOptimal.

When MoveCursors() is forced to make a physical cursor move, it picks the "best" virtual cursor in the subtree rooted at $q$ to move (line 9). This is essentially the cursor that is predicted to move the furthest. How to determine the best cursor to move is beyond the scope of this papers. For various heuristics see [10].

MoveCursorsBottomUp(), which is shown in Figure 5 recursively performs a bottom-up pass over the subtree under consideration. The goal of this pass is to try and move each parent cursor forward so it contains its children cursors. If $q$ is an AND node (line 4), then $C q$ must contain the cursor of its max child in order for there to be an extension. Similarly, if $q$ is an OR node (line 8), then $C q$ must contain the cursor of its min child. Finally, if $q$ is a path node with a child (line 12), then $C q$ must contain that child's cursor.

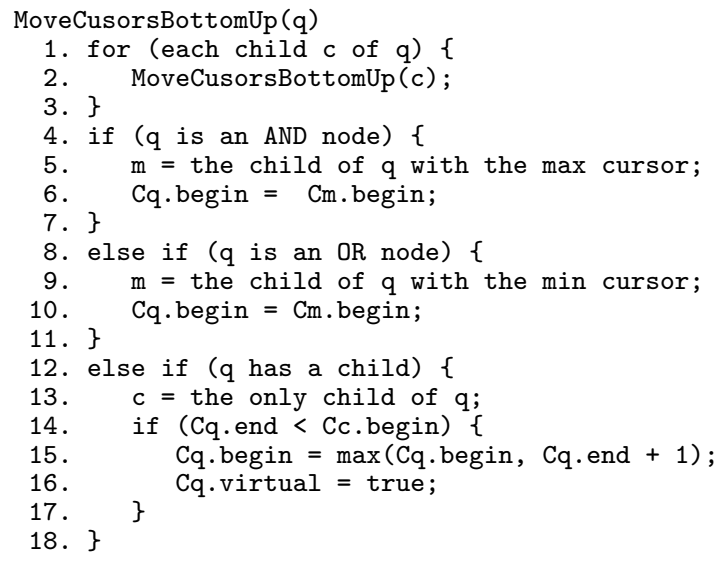

Figure 5: The bottom-up pass to move cursors
Recall that each branching node's cursor is used to pass along the position of its parent cursor or a child cursor. Here, in the case of an AND node, $C q$ is used to pass up the begin value of its max child cursor (lines 5-6). Similar action is taken in the case of an OR node with its min child cursor (lines 9-10). Finally, if $q$ is a path node with a child, and Cq.end falls before that child's cursor, then $C q$ is virtually moved to the max of Cq.begin or Cq.end +1 (lines 15-16), which is the most $C q$ can be moved forward without missing an extension. The max is needed (line 15) to deal with the case where a previous call to MoveCursors has already virtually moved $C q$.begin past $C q . e n d$.

After MoveCursorsBottomUp() finishes, each cursor will have been virtually moved as far forward as its children cursors will allow it to be moved without missing an extension. MoveCursorsTopDown(), which is shown in Figure 6, is then called to recursively perform the top-down pass over the subtree under consideration. The goal of this pass is to try and move each child cursor forward so it is contained by its parent cursor.

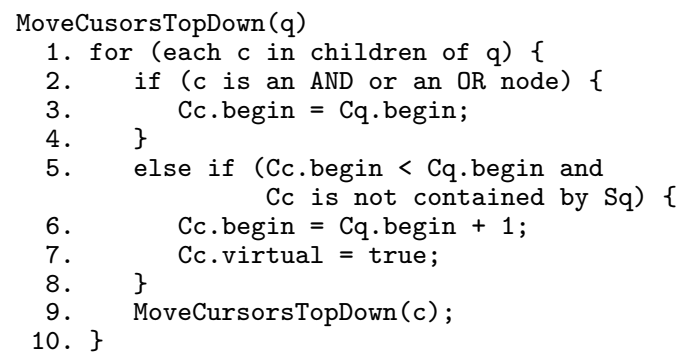

Figure 6: The top-down pass to move cursors

In MoveCursorsTopDown(), $c$ and $q$ correspond to the current child and parent nodes being examined, respectively. If $c$ is a branching node, then the child cursor $C c$ is used to pass down the position of the parent cursor $C q$ (line 3 ). Else, if $C$ c.begin falls before $C q$ and is not contained by its parent stack $S q$, then $C c$ is virtually moved to $C q$.begin +1 (lines 5-8), which is the most $C c$ can be moved forward without missing an extension. $S q$ needs to be checked for containment in this case to guard against missing solutions when there is recursive data for $q$.

\subsection{Outputting a Solution}

Solutions are output and pushed onto the stacks in OutputAndPush(), which is shown in Figure 7. Before the cursor of node $q$ is stacked (line 8 ), a check is made to see if $q$ corresponds to the root of $Q$. If so, then one or more solutions are output before the new root cursor is stacked (lines 2-6).

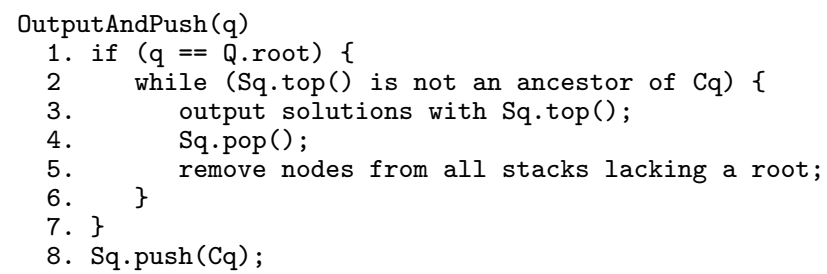

Figure 7: Outputting a solution

The simple stacking method use here is not optimized for space, since that is not the focus of this paper. However, note that OutputAndPush() can easily be changed to use the stacking method described in [10], which is optimized along those lines. 


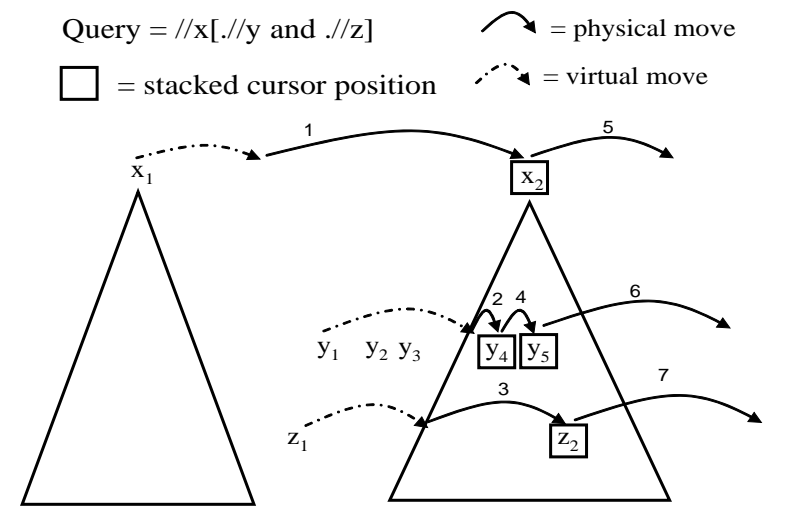

Figure 8: Cursor movement example

\subsection{Cursor Movement Example}

We now illustrate TwigOptimal's cursor movement using the query shown in Figure 8. In this example, there are three cursors on posting lists, one per query tree node. The scope of each $x$ element is represented by a triangle enclosing its descendant $y$ and $z$ elements. For clarity, the figure does not show a complete XML document, but rather only two sibling $x$ elements. The left-to-right position of the letters in the figure indicates the relative position of the data nodes in the document. Physical cursor moves are depicted by solid curved lines, labeled with the sequence number in which they are performed, while virtual moves are depicted by dashed lines.

At the beginning, the three cursors are positioned on $x_{1}$, $y_{1}$, and $z_{1}$, which do not form an extension. $C x$ is the min cursor and there is no extension for it, so MoveCursors $(x)$ is called. Since $x_{1}$ ends before $y_{1}$ and $z_{1}, C x$ is virtually moved just past the end of $x_{1}$. At that point, $C x$ is still the min cursor, so it is physically moved to $x_{2}$, after which $C z$ becomes the min cursor. In the subsequent calls to MoveCursors(), $C z$ and $C y$ are virtually moved within $x_{2}$, causing postings $y_{2}$ and $y_{3}$ to be skipped. Then $C y$ is physically moved to $y_{4}$. At that point $C x$ is the min cursor again, but the call to Extension $(x)$ returns false since $C z$ is virtual. $C z$ is then physically moved to $z_{2}, C x$ remains the min cursor, and the next call to Extension $(x)$ succeeds.

The call to OutputAndPush $(x)$ stacks $x_{2}$ and then $C x$ is physically moved to the next $x$ (not shown). At that point, $C y$ is the min cursor and, since Extension(y) returns true, $y_{4}$ is stacked and physically moved to $y_{5}$. The same steps are repeated for $y_{5}$ since it is also part of an extension. $C y$ is then physically moved to the next $y$ (not shown). This ensures that all the $y$ nodes within $x_{2}$ have been exhausted. Finally, $C z$ becomes the min cursor, Extension(z) returns true, and $z_{2}$ stacked. Since $C z$ is still the min cursor, it is moved again, this time beyond $x_{2}$. At that point, there are four stack entries that make up two solutions. These will be output after the next $x$ node is stacked.

\section{COMPARISON TO EXISTING ALGORITHMS}

Figure 9 and Figure 10 are used to illustrate how TwigOptimal can generate far fewer physical cursor moves than existing twig join algorithms. Both figures trace the evaluation of the query $/ / \mathrm{w} / / \mathrm{x} / / \mathrm{y} / / \mathrm{z}$ on two sibling $w$ elements. Figure 9 illustrates how the cursors would be moved using the twig join algorithm described in [10], which represents the

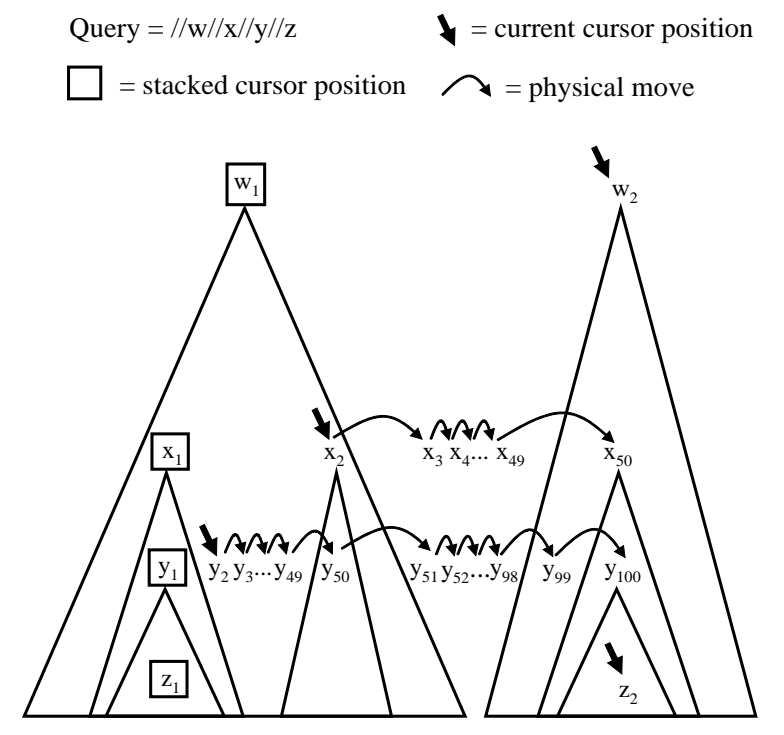

Figure 9: Counting physical cursor moves with existing algorithms

state of the art among existing algorithms, while Figure 10 illustrates how the cursors would be moved in TwigOptimal.

As shown, both algorithms start out in the same state, with the first solution $w_{1}, x_{1}, y_{1}$ and $z_{1}$ stacked, and with $C w, C x, C y, C z$ positioned on $w_{2}, x_{2}, y_{2}$ and $z_{2}$, respectively. We compare the number of physical cursor moves needed for each algorithm to reach the second solution, which is $w_{2}, x_{50}, y_{100}$ and $z_{2}$.

In $[10]$, when the test for an extension on $y$ returns false, a "broken edge" in the subtree rooted at $y$ is identified. A broken edge is a parent-child pair, where the parent cursor does not contain its child cursor. The parent and child cursors for the broken edge are then repeatedly moved in a coordinated way until the parent cursor contains the child cursor. As shown in Figure 9, this causes $C x$ and $C y$ to be moved between $x_{2}-x_{50}$ and $y_{1}-y_{100}$, respectively, resulting in roughly 150 physical cursor moves to find the second solution.

In contrast, as shown in Figure 10, TwigOptimal performs only two physical cursor moves. Three virtual moves place $C x$ and $C y$ within $w_{2}$, and then two physical cursor moves are made to find the second solution.

\section{OPTIMALITY PROOF SKETCH}

In this section, we provide a sketch of TwigOptimal's optimality. Our main claim is stated in Theorem 1.

THEOREM 1. Given an evaluation state $\langle Q, C, S>$, whenever a cursor is physically moved in TwigOptimal:

1. It is necessary to move one of the cursors considered by TwigOptimal.

2. The cursor that is physically moved is moved as far forward as possible without missing a solution.

For the first part of Theorem 1, we examine the two places in TwigOptimal where forwardTo() is called to physically move a cursor. In ExecuteQuery(), a cursor $C q$ is physically moved after $q$ has been found to be a part of an extension (and thus, a solution). This physical move is necessary to 


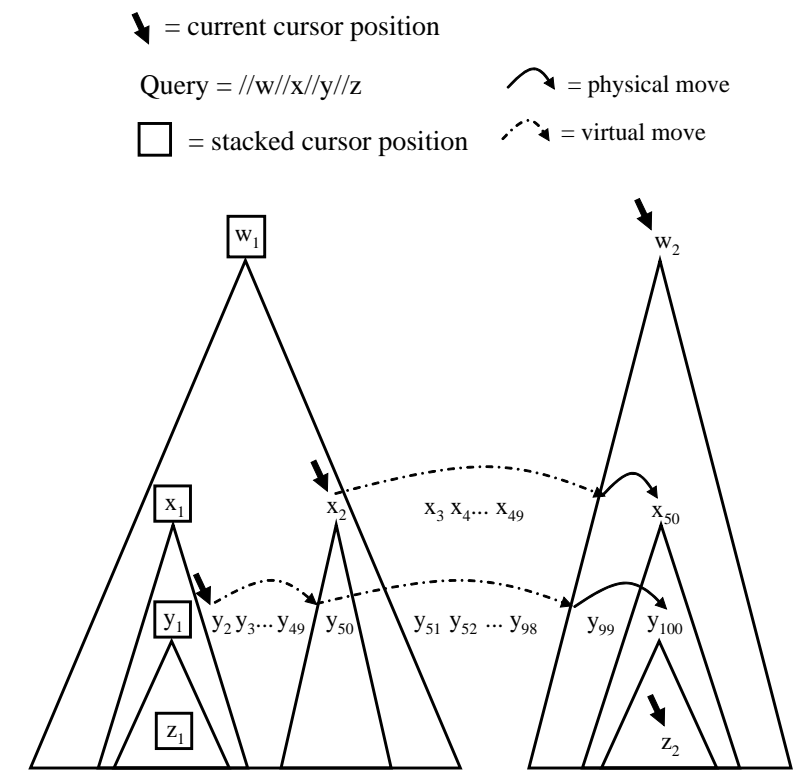

Figure 10: Counting physical cursor moves with TwigOptimal

check if $C q$ 's next position is part of a solution. For example, in Figure 8, $C y$ has to be physically moved until it exits from the $x_{2}$ 's subtree. If any other cursor is moved at that point, the solution $x_{2}, y_{5}$ and $z_{2}$ would be missed.

The second call to forwardTo() is performed in MoveCursors() when the min cursor is virtual. In that case, the two passes over the query tree have virtually moved cursors in the subtree of the min cursor. If some of the cursors are virtual, TwigOptimal cannot confirm that it has found an extension. Therefore, TwigOptimal has to physically move one of the virtual cursors to find out if it has found a solution. This is illustrated in Figure 8 when $C z$ is moved to check if there is a $z$ posting within $x_{2}$.

For the second part of Theorem 1, it can be shown by induction that the two-pass algorithm used in MoveCursors() passes along restrictions on the maximum possible location of the next physical cursor move from descendants to ancestors (in the bottom-up pass) and then from ancestors to descendants (in the top-down pass). Therefore, after MoveCursors() has been called, the cursors will have been moved as far forward as possible without missing a solution.

Note that if more than one virtual cursor is available to be physically moved, TwigOptimal chooses the "best" cursor using heuristics based on the available statistics [10]. This means that TwigOptimal is not instance optimal [6], as instance optimality can only be achieved if there was a way to always choose the best cursor to move. However, MoveCursors() does guarantee that when a cursor is physically moved, it is moved as far forward as possible without missing a solution.

Also note that Theorem 1 assumes forward-only cursor moves on a standard inverted index. Unfortunately, this means that when an ancestor cursor is moved to contain a descendant cursor in MoveCursorsBottomUp(), TwigOptimal can behave inefficiently. Special indexes and the cursor method forwardToAncestor() have been proposed to deal with this situation $[8,13]$. Instead of using a special index, we propose an implementation of forwardToAncestor() that simply moves the ancestor cursor forward to the descen- dant cursor, then backs up the ancestor cursor to the first position before the descendant cursor. In the (rare) case of recursion, the backward step may have to be repeated. If forwardTo() is modified to detect when to call forwardToAncestor() under the covers, then nothing in TwigOptimal has to be changed to take advantage of this optimization.

TheOREM 2. Assuming forward-only cursor moves, TwigOptimal performs the minimum number of physical cursor moves to evaluate $Q$.

The proof follows from Theorem 1, where we conclude that every physical cursor move performed by TwigOptimal is necessary and goes as far forward as possible without missing a solution.

\section{EXTRACTION POINTS}

Existing twig join algorithms assume that all path nodes in a query tree need to be output. However, an XQuery return clause often requires only a subset of the path nodes being matched to be output. For example, in Figure 1, only the title node needs to be output. By being aware of these extraction points and "skipping" over data nodes that do not need to be output, the number of cursor moves performed by a twig join can be dramatically reduced. This section describes how extraction points can be used to improve TwigOptimal's performance by making only minor changes to ExecuteQuery(), as shown in Figure 11. Lines 9a-9j in Figure 11 simply replace line 9 in Figure 2.

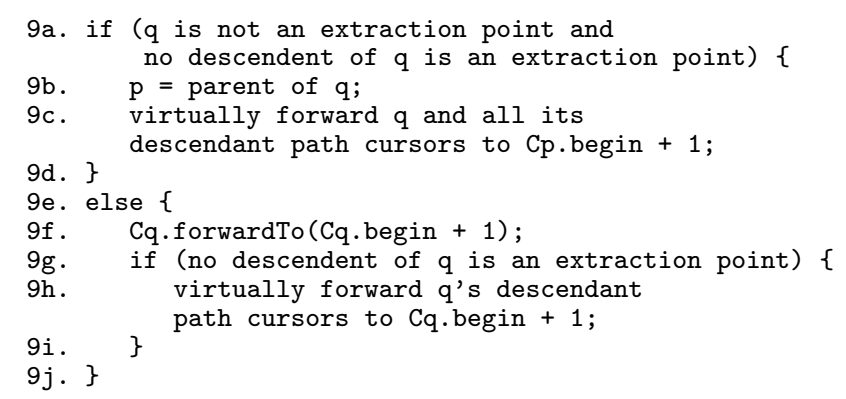

Figure 11: Changes to ExecuteQuery() for extraction points

To understand the code in Figure 11, let $q$ be a path node in the query tree with parent $p$ and consider the four possibilities for $q$ and its descendants:

1. Neither $q$ nor any of its descendants in the query tree are extraction points.

2. Node $q$ is an extraction point but none of its descendants are extraction points.

3. Both $q$ and some descendant of $q$ are extraction points.

4. Node $q$ is not an extraction point but some descendant of $q$ is an extraction point.

Case 1 ) is handled by lines $9 \mathrm{a}-9 \mathrm{~d}$. In this case, it should be clear that TwigOptimal does not need to find all the extensions rooted at $q$. Consequently, once an extension for $q$ has been found, $C q$ and all its descendant cursors can be virtually moved within $C p$ (line 9c).

Case 2) is handled by lines $9 \mathrm{e}-9 \mathrm{i}$. In this case, it should be clear that TwigOptimal does not need to find all the extensions below $q$. Consequently, after physically moving $C q$ (line 9f), all its descendant cursors can be virtually moved to within $C q$ (line $9 \mathrm{~h}$ ). 


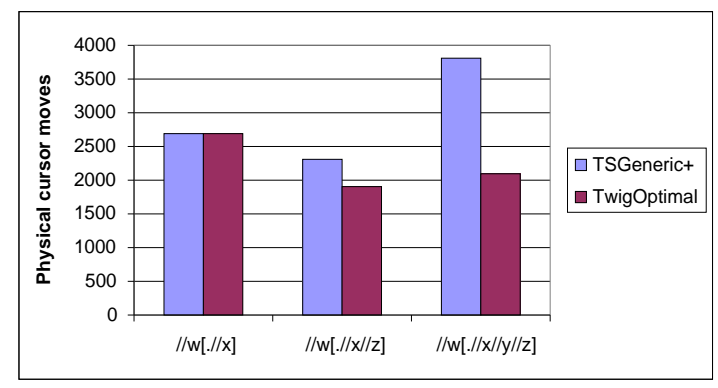

Figure 12: Physical cursor moves for the synthetic dataset

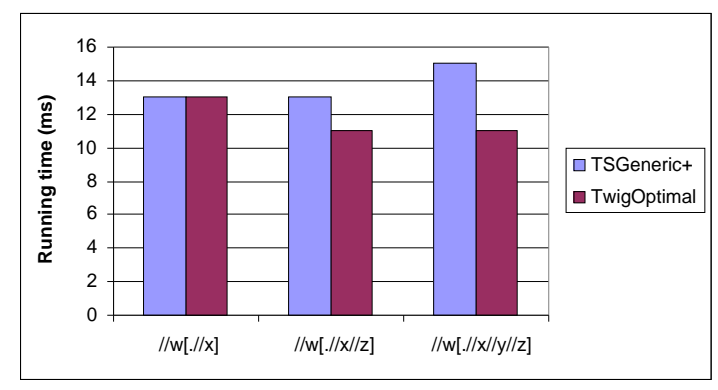

Figure 13: Running time for the synthetic dataset

Finally, case 3) and case 4) fall through the first part of the if-statement and are handled the same as in the version of ExecuteQuery() without extraction points, that is, just line $9 \mathrm{f}$ is executed. Also, although it is not shown, OutputAndPush() changes with extraction points. In particular, if neither $q$ nor any of its descendants are extraction points, then $C q$ should not be stacked.

\section{EXPERIMENTAL RESULTS}

In this section, we present experimental results comparing TwigOptimal with TSGeneric + [9, 10], which represents the state of the art among existing twig join algorithms, Results are also provided to show how TwigOptimal's performance can be improved by being aware of extraction points.

Our experimental testbed was built on the Berkeley DB [14] embedded database, where we implemented both TwigOptimal and TSGenerict. All experiments were run on a Linux Red Hat 8.0 workstation, with a $2.2 \mathrm{GHz}$ Intel Pentium 4 processor and 2 GB of main memory. Although, the amount of main memory was large, both twig join algorithms only access their posting lists in the forward direction (with skips). Consequently, our running times are representative of any memory buffer large enough to hold at least the internal pages of the B-tree and one leaf page per posting list.

For data, we used the XMark [17] dataset and a synthetic dataset [18]. For both datasets, the difference in the relative performance of TwigOptimal and TSGeneric+ was similar.

\subsection{Results without Extraction Points}

In all our experiments, TwigOptimal and TSGeneric+ both used the same top-down strategy [10] for picking which cursor to physically move next. As noted earlier, if both algorithms use the same strategy for picking which cursor to move next, TwigOptimal should never generate more phys-

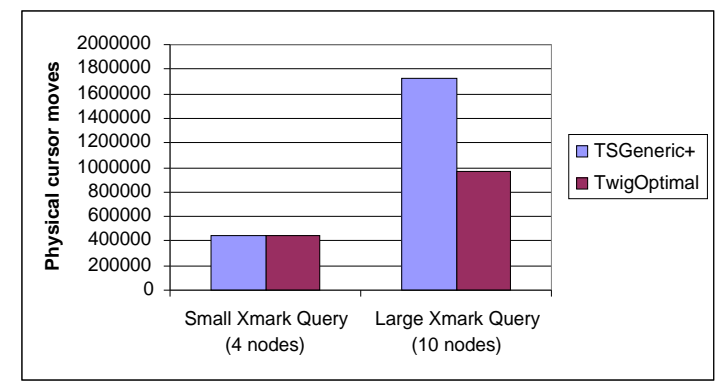

Figure 14: Physical cursor moves for the XMark dataset

ical cursor moves than TSGenerict. Moreover, by looking more globally at the query's evaluation state to determine which cursor to move next, TwigOptimal should be able to generate fewer physical cursor moves in many cases.

Figure 12 shows the number of physical cursor moves for three queries on the synthetic dataset, while Figure 13 shows their running time using cold buffers. As shown, both algorithms performed exactly the same on the first query. This is because the query tree in that case only had two levels (i.e., two cursors), providing little room for TwigOptimal to optimize its cursor movement. However, as the queries got more complex and the number of levels in their query tree grew, TwigOptimal generated $40 \%$ fewer physical cursor moves than TSGeneric + and its running time improved proportionally.

For the XMark dataset, we studied two queries, a small one with a 4-node query tree, and a large one with a 10-node query tree:

- //item//description["science" and "paper"]

- //item//description//parlist//listitem//text ["science" and "logotype" and "benefit" and "paper" and "windsor"]

Figure 14 shows the number of physical cursor moves for these queries. As with the synthetic dataset, on the smaller query, both algorithms generated roughly the same number of cursor moves, while for the larger query, TwigOptimal generated $45 \%$ fewer cursor moves. TwigOptimal's running time also improved proportionally, but because of space limitations, the graph with those results has been omitted.

\subsection{Results with Extraction Points}

Figure 15 and Figure 16 show how TwigOptimal's performance can be improved by being aware of extraction points. The graphs in those figures were generated using the synthetic dataset. Moving from left to right in the graphs, the number of extraction points in the query decreases from all 4 nodes to 1 node. The left-most query represents TwigOptimal without any optimization for extraction points.

As expected, the number of physical cursor moves TwigOptimal made decreased as the number of extraction points in the query decreased. The fact that they decreased somewhat linearly is an artifact of the dataset and the way it was generated with uniform probabilities. In general, the impact of extraction points on performance can vary dramatically, depending on the query and the dataset. The improvement in performance can be substantial anytime there is a pattern like $\mathrm{a}[. / / \mathrm{b}]$, where there are a large number of $b$ nodes under each $a$ node. To illustrate this, another data point was taken from the XMark dataset. On that dataset, the query //item//text with 2 extraction points generated 


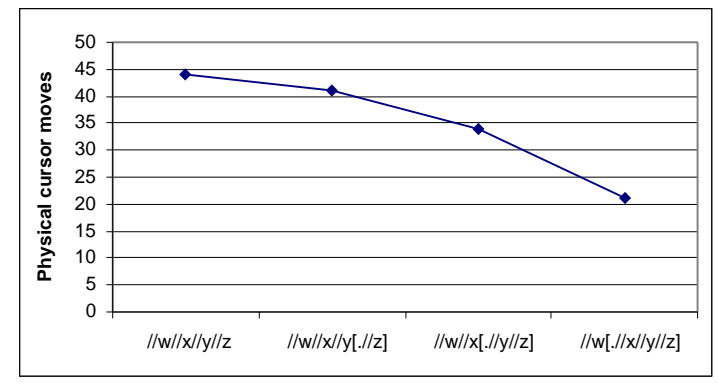

Figure 15: Physical cursor moves for queries with different number of extraction points

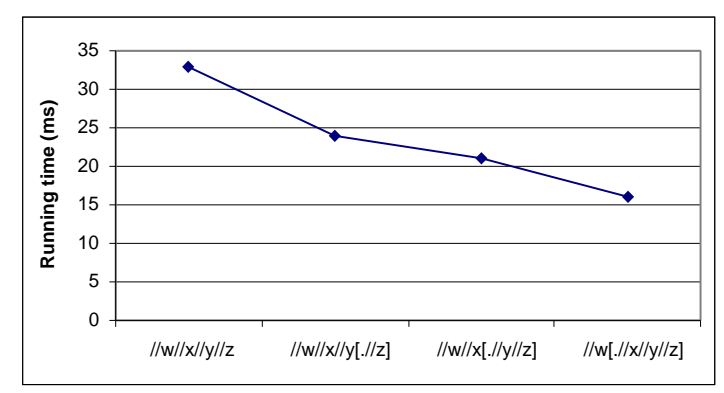

Figure 16: Running time for queries with different number of extraction points

835,740 cursor moves, whereas the same query with 1 extraction point //item[.//text] generated 435,000 cursor moves, roughly half as many.

\section{CONCLUSION}

Because each cursor move can trigger I/O, the performance of a holistic twig join is largely determined by how many cursor moves it makes. Yet, existing twig join algorithms have not been optimized along these lines. Instead, they make a local, and hence sub-optimal, decision in choosing which cursor to move next and how far to move it. In this paper, we described TwigOptimal, a new holistic twig join algorithm that minimizes the number of cursor moves it makes by looking more globally at the query's state to determine which cursor to move next. Experimental results were provided, showing that TwigOptimal can outperform existing twig joins algorithms by up to $40 \%$.

Another shortcoming of existing twig join algorithms is that they assume all nodes in a query tree need to be output. However, an XQuery return clause often requires only a subset of the nodes being matched to be output. By being aware of these so-called extraction points, we described how TwigOptimal is able to further reduce the number of cursors moves it makes. Experimental results were provided, showing that this can provide a substantial improvement in performance.

\section{REFERENCES}

[1] S. Al-Khalifa, H. Jagadish, N. Koudas, J. Patel, D. Srivastava, and Y. Wu. Structural joins: A primitive for efficient $\mathrm{xml}$ query pattern matching. In $I C D E, 2002$.

[2] Z. Bar-Yossef, M. Fontoura, and V. Josifovski. Buffering in query evaluation over xml streams. In PODS, 2005.

[3] N. Bruno, N. Koudas, and D. Srivastava. Holistic twig joins: Optimal xml pattern matching. In SIGMOD, 2002.

[4] T. Chen, J Lu, and T. W. Ling. On boosting holism in xml twig pattern matching using structural indexing techniques. In SIGMOD, 2005.

[5] S. Chien, Z. Vagena, D. Zhang, V. Tsotras, and C. Zaniolo. Efficient structural joins on indexed xml documents. In $V L D B, 2002$.

[6] R. Fagin, A. Lotem, and M. Naor. Optimal aggregation algorithms for middleware. Journal of Computer and System Sciences, 66(4):614-656, 2003.

[7] G.Salton and M. J. McGill. Introduction to modern information retrieval. McGraw-Hill, 1983.

[8] H. Jiang, H. Lu, W. Wang, and B. C. Ooi. Xr-tree: Indexing $\mathrm{xml}$ data for efficient structural join. In ICDE, 2003.

[9] H. Jiang, W. Wang, and H. Lu. Efficient processing of xml twig queries with or-predicates. In SIGMOD, 2004.

[10] H. Jiang, W. Wang, H. Lu, and J. Yu. Holistic twig joins on indexed xml documents. In $V L D B, 2003$.

[11] V. Josifovski, M. Fontoura, and A. Barta. Querying xml streams. In VLDB Journal, 14(2), 2005.

[12] R. Kaushik, R. Krishnamurthy, J. Naughton, and R. Ramakrishnan. On the integration of structure indexes and inverted lists. In SIGMOD, 2002.

[13] Q. Li and B. Moon. Indexing and querying xml data for reguar path expressions. In $V L D B, 2001$.

[14] M. Olson, K. Bostic, and M. Seltzer. Berkeley DB. In Summer Usenix Technical Conf., 1999.

[15] I. Tatarinov, S. Viglas, K. Beyer, J. Shanmugasundaram, E. Shekita, and C. Zhang. Storing and querying ordered xml using a relational dbms. In SIGMOD, 2002.

[16] H. Wang, S. Park, W. Fan, and P. Yu. Vist: A dynamic index method for querying $\mathrm{xml}$ data by tree structures. In SIGMOD, 2003.

[17] Xmark: The xml benchmark project. http://monetdb.cwi.nl/xml/index.html.

[18] B. Yang, M. Fontoura, E. Shekita, S. Rajagopalan, and K. S. Beyer. Virtual cursors for xml joins. In CIKM, 2004.

[19] C. Zhang, J. Naughton, D. DeWitt, Q. Luo, and G. Lohman. On supporting containment queries in relational database management systems. In SIGMOD, 2001 